ARTICLE

\title{
Upcycling of dynamic thiourea thermoset polymers by intrinsic chemical strengthening
}

\author{
Haijun Feng ${ }^{1,2}$, Ning Zheng (10 ${ }^{1 凶}$, Wenjun Peng ${ }^{1}$, Chujun Ni ${ }^{1}$, Huijie Song ${ }^{1}$, Qian Zhao (i] ${ }^{1,2} \&$ Tao Xie (i] ${ }^{1,2}$
}

Thermoset polymers are indispensable but their environmental impact has been an everincreasing concern given their typical intractability. Although concepts enabling their reprocessing have been demonstrated, their practical potential is limited by the deteriorated performance of the reprocessed materials. Here, we report a thiourea based thermoset elastomer that can be reprocessed with enhanced mechanical properties. We reveal that the thiourea bonds are dynamic which leads to the reprocessibility. More importantly, they can undergo selective oxidation during high temperature reprocessing, resulting in significant chemical strengthening within certain reprocessing cycles. This is opposite to most polymers for which reprocessing typically results in material deterioration. The possibility of having materials with inherent reprocessing induced performance enhancement points to a promising direction towards polymer recycling.

\footnotetext{
${ }^{1}$ State Key Laboratory of Chemical Engineering, College of Chemical and Biological Engineering, Zhejiang University, 38 Zheda Road, Hangzhou 310027, P. R. China. ${ }^{2}$ ZJU-Hangzhou Global Scientific and Technological Innovation Center, Hangzhou 311215, P. R. China. ${ }^{凶}$ email: zhengning@zju.edu.cn
} 
T he discovery of synthetic polymers has brought enormous benefits to the human society. However, their excessive use has led to concern on the negative impact to the environment ${ }^{1}$. Thermoplastic polymers can be reprocessed ${ }^{2}$, but their performance is generally reduced, limiting their practical value. By comparison, classical thermosets are not reprocessible due to their permanent cross-linked nature. Despite such, thermosets remain indispensable given their unique advantages (e.g., dimension stability) over thermoplastic polymers ${ }^{3}$. Thermoset rubbers in particular have been widely used in many aspects of our daily lives including tires, sealants, adhesives, and medical devices. To mitigate the negative environmental effect of thermosets, extensive efforts have been made to develop new technologies to recycle them ${ }^{4-8}$. A particularly attractive approach is to make them reprocessible by introducing dynamic covalent bonds into the networks ${ }^{9-21}$ or activating the dynamic characteristics of the otherwise conventional covalent bonds with catalysts ${ }^{22-25}$. Despite the promising developments at the experimental scale, successful implementation at the commercial level has been quite limited (if any). One major reason is that reprocessed thermosets typically experience unavoidable mechanical performance deterioration. During reprocessing, three destructive molecular events generally happen: chain scission, degradation, and oxidation. The first one is due to the encountering of large mechanical forces (e.g., grinding) whereas the latter two originate from the exposure to high temperature under realistic conditions in which oxygen/water is present. In principle, better mechanical recovery is possible if side reactions could be alleviated. Indeed, for a thioureathane network, introducing excess thiol groups was shown to effectively counter the side reaction between isocyanates and environmental water, leading to full mechanical recovery even after three reprocessing cycles ${ }^{24}$. However, the above approach does not allow upcycling for which the reprocessed materials can show better mechanical properties than the original material.

In this work, we hypothesize that a more effective approach is to develop a proactive mechanism that can counteract all the side effects that lead to mechanical deterioration. Such an approach potentially can even make the reprocessed materials stronger than the original ones. Hereafter, we describe our successful attempt in this direction with a dynamic thiourea-based thermoset elastomer. Our original motivation of exploring thiourea bonds is two folds: thiourethane bonds are more readily dynamic than urethane bonds ${ }^{24}$; urea and hindered urea bonds are both dynamic with the steric hindrance in the latter enhancing the dynamic exchangeability ${ }^{26}$. We emphasize that non-cross-linked thiourea polymers are known to exhibit unusual room temperature selfhealing behavior via their unique zig-zag hydrogen bonds ${ }^{27}$, but such a principle is different from the utilization of the dynamic covalent characteristics for designing reprocessable thermosets.

\section{Results}

Polymer network design and the principle for strengthening. The enabling chemistry is shown in Fig. 1a. The network is synthesized by reacting thiocarbonyldiimidazole with a primary diamine and a secondary diamine, along with a triamine crosslinker. The curing reaction proceeds at $140^{\circ} \mathrm{C}$ under nitrogen flow without any catalyst, through a substitution reaction ${ }^{27}$ with the byproduct imidazole removed by the gas flow. Two types of thiourea bonds are produced, namely, a non-hindered thiourea from the primary amine and a hindered thiourea from the secondary amine. We note here that linear polymers containing thiourea bonds have been reported in the literature ${ }^{27,28}$, but their dynamic covalent nature is not known. Our discovery here is that the thiourea bonds are dynamic covalent bonds. The other discovery is that the hindered thiourea bonds can undergo controllable thermal oxidation in air to yield the corresponding urea. As illustrated in Fig. 1b, the dynamic thiourea exchange reaction enables the reprocessing of the cross-linked polymer. Surprisingly, the conversion from the hindered thiourea to the urea as the result of the otherwise undesirable thermal oxidation yields a strengthening effect, making the reprocessed polymer stronger than the original one.

Dynamic property of thiourea network. The dynamic nature of the thiourea bond is verified through an exchange reaction conducted with 1-hexyl-3-isopropyl-thiourea as the small molecular model compound. Its self-exchange reaction is expected to yield 1,3-diisopropylthiourea and 1,3-dihexylthiourea (Fig. 2a), which is confirmed by gas chromatographic analyses presented in Supplementary Fig. 1. Detecting 1,3-diisopropylthiourea by gas chromatography therefore allows monitoring the exchange reaction. Accordingly, Fig. 2a shows that heating promotes the exchange reaction until reaching the equilibrium states at about one hour between 130 and $150^{\circ} \mathrm{C}$. The activation energy for the thiourea exchange is determined as $69.1 \mathrm{~kJ} / \mathrm{mol}$ (Supplementary Fig. 1).

Having verified the dynamic thiourea exchangeability with the small molecule, we proceed to investigate their impact on polymer networks. A series of cross-linked polymers (detailed formulations in Supplementary Table 1) are synthesized with an identical cross-linking density but variation in the molar percent of the hindered thiourea with respect to the total thioureas. These samples are subjected to isostrain stress relaxation experiments at $130^{\circ} \mathrm{C}$. The results (Fig. 2c) show that all of them exhibit complete stress relaxation. The stress relaxation is faster for samples with higher amounts of the hindered thiourea, suggesting that the steric hindrance promotes faster exchange, similar to the trend known for urea bonds ${ }^{28-30}$.

The hindered thiourea bond in the network affects the mechanical properties of the materials. As its molar ratio increases from 0 to $34 \%$ (Fig. $2 \mathrm{~d}$ ), the modulus decreases linearly between 1.25 and $0.51 \mathrm{MPa}$. By comparison, its elongation at break raises gradually within a relatively narrow range between 152 and $190 \%$. Unless otherwise noted, we hereafter choose the polymer sample with $21 \%$ hindered thiourea bonds (light red rectangle in Fig. 2d) for further investigation. The sample does not dissolve but swells in a range of solvents (gel content: $83 \pm 5 \%$, see Supplementary Table 2), confirming its cross-linked nature. In addition, the sample is also chemically stable against acidic and basic $\mathrm{N}, \mathrm{N}$-dimethylformamide. Nevertheless, Fig. 2e illustrates that this sample can undergo faster stress relaxation at higher temperatures with a corresponding activation energy of $115.8 \mathrm{~kJ} / \mathrm{mol}$ calculated from the inset figure. Complete stress relaxation is reached at a relatively short time of $15-20 \mathrm{~min}$ at $140^{\circ} \mathrm{C}$, which is chosen as the appropriate reprocessing temperature. We emphasize here that the sample is a fully reacted network since further thermal posttreatment under nitrogen does not result in any change to the material, including the composition, mechanical and thermal properties (Supplementary Fig. 2 and Fig. 3a). This is a critical reference for the thermal oxidation enhanced mechanical properties presented later in the context.

Selective oxidation of the hindered thiourea bond. As illustrated in Fig. 1, the most critical concept here is that the dynamic network can not only be reprocessed, but also undergo constructive thermal oxidation to enhance the mechanical properties. To verify the thermal oxidation reaction, a hindered thiourea (Supplementary Fig. 3) is chosen as a small molecular model compound. It is exposed to an oxygen atmosphere at $140{ }^{\circ} \mathrm{C}$. 
a<smiles>CCNCCCCCCNCCN</smiles>

Curing reaction

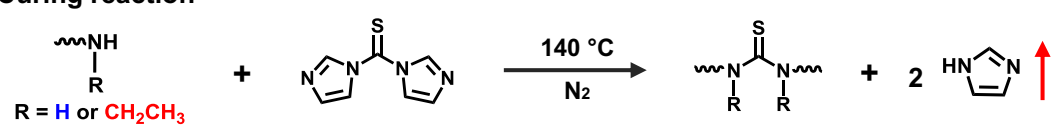

Dynamic exchange

Thermal oxidation
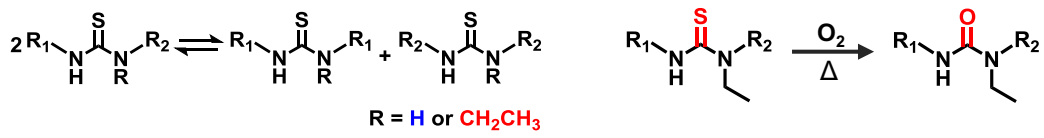

b

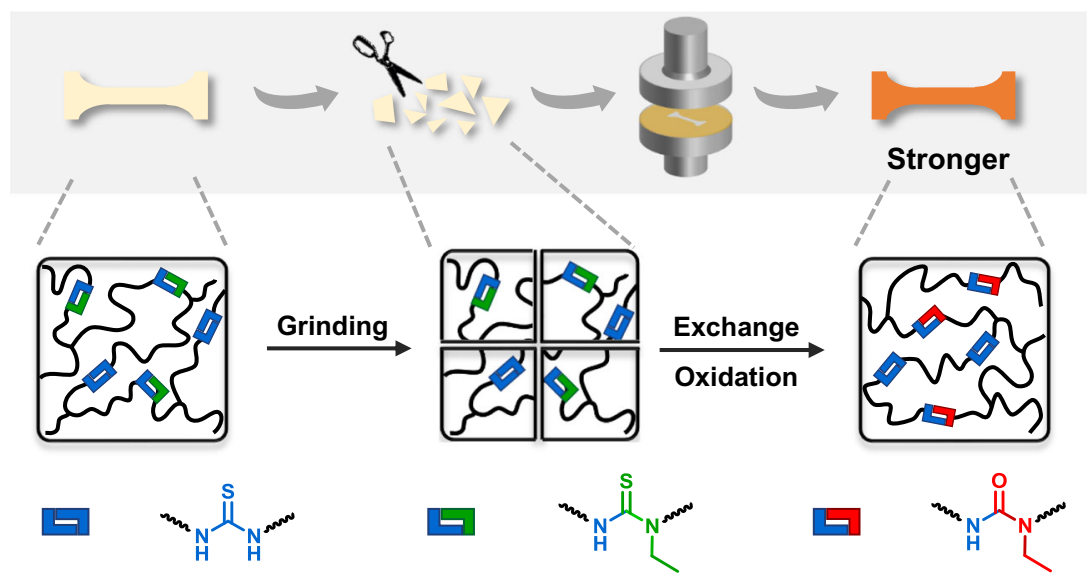

Fig. 1 Polymer network design and the principle for strengthening. a The chemistry for network synthesis, reprocessing, and oxidation. b Schematic illustration of reprocessing and strengthening of the cross-linked network.

${ }^{1} \mathrm{H}-\mathrm{NMR}$ (Supplementary Fig. 3) of the oxidized products in comparison with the original compound suggest that hindered urea is indeed progressively produced. HPLC-MS analysis (Supplementary Fig. 4) further confirms that the oxidation converts the hindered thiourea into the corresponding urea, along with some side products corresponding to a molecular weight increment of 14 (the molecular weight of methylene) from these two compounds. We note here that HPLC-MS is only qualitative since the sensitivity of each compound is unknown. Nevertheless, the ${ }^{1} \mathrm{H}$ NMR (Supplementary Fig. 3 ) suggests that these side products are minor compared to the main oxidation product (i.e., urea). In the infrared analyses (Fig. 3b), the appearance of a urea characteristic band at $1640 \mathrm{~cm}^{-1}$ after oxidation further verify the oxidation reaction. By comparison, an unhindered thiourea is found to be stable under the same oxidation conditions (Supplementary Fig. 5). From the mechanism standpoint, the conversion from thiourea to urea follows two steps: (1) oxygen forms an intermediate complex with the thiourea; (2) the complex loses sulfur monoxide to form urea ${ }^{31}$.

The oxidation-induced conversion from the hindered thiourea to the urea is found to significantly enhance the mechanical performance of the network polymer. The stress-strain curves of the synthesized sample before and after oxidation for $48 \mathrm{~h}$ in air at $140{ }^{\circ} \mathrm{C}$ (without reprocessing) are presented in Fig. 3c. The original material has a modulus of $0.74 \mathrm{MPa}$, strength of $0.96 \mathrm{MPa}$, respectively. After the oxidation, the modulus and strength are markedly increased to 2.06 and $2.88 \mathrm{MPa}$, while the thermal treatment in nitrogen hardly make any differences in mechanical properties (Fig. 3a). Notably, even the maximum strain is raised from 192 to $210 \%$ after oxidation. The strengthening of the all-around mechanical performances is surprising as common knowledge suggests that polymer oxidation usually leads to deterioration of mechanical properties (e.g., becoming more brittle). We believe such a desirable effect arises from the ability of thiourea and urea to form hydrogen bonds of different strengths. Compared with the sulfur atom, the oxygen atom in urea has higher electronegativity and smaller atomic radius, with both attributes favoring stronger hydrogen bonds ${ }^{32}$. The existence of hydrogen bonds in both the original and reprocessed samples (with $24 \mathrm{~h}$ of oxidation) is verified by the temperature ramping FTIR analysis. The results (Supplementary Fig. 6) show the weakening of hydrogen bonding $\left(3299 \mathrm{~cm}^{-1}\right)$ upon heating accompanied by the appearance of free $\mathrm{N}-\mathrm{H}$ bond $\left(3420 \mathrm{~cm}^{-1}\right)$ and such a trend is reversed upon cooling. We note that, while the network polymer can be oxidized at elevated temperatures, it is stable under ambient condition. Its mechanical properties remain largely unchanged after being kept in ambient air for 60 days (Supplementary Fig. 7).

Network reprocessing and enhancement in mechanical properties. The dynamic characteristics of the thiourea bonds, along with the favorable oxidation chemistry, establishes an unusual basis for a cross-link polymer to be reprocessed into a stronger material. For reprocessing, the polymer is ground into small pieces and mold-pressed ( $4 \mathrm{MPa}, 30 \mathrm{~min}, 140^{\circ} \mathrm{C}$ ) (Fig. 4a). To harness the benefit of the oxidation, an important conditioning step $\left(140^{\circ} \mathrm{C}\right.$ in open air) is performed after the pressure molding.

The impact of this chemical change on the mechanical properties of the reprocessed polymer are demonstrated in Fig. $4 \mathrm{~b}$ and Supplementary Fig. 8. Without conditioning (conditioning 
a<smiles>[Z]CCCCCNC(=S)NC(C)C=C</smiles>

b

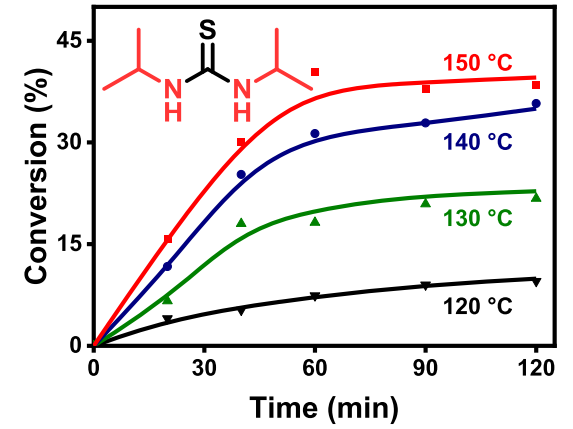

d

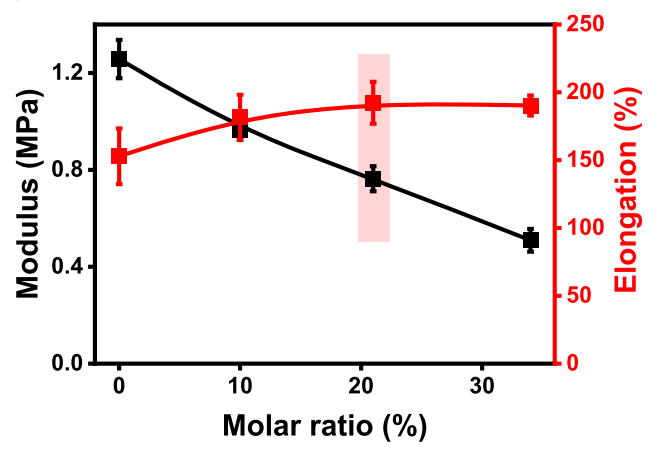

C

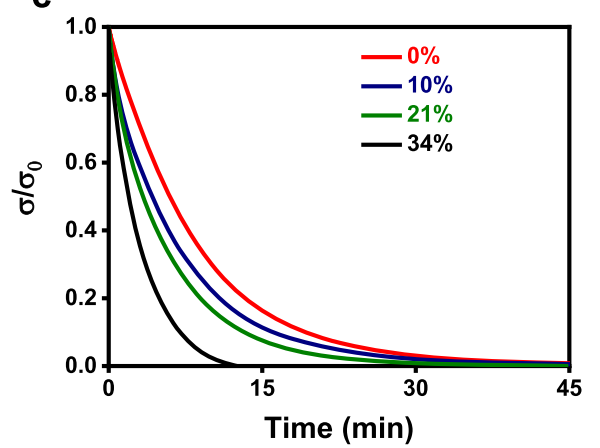

e

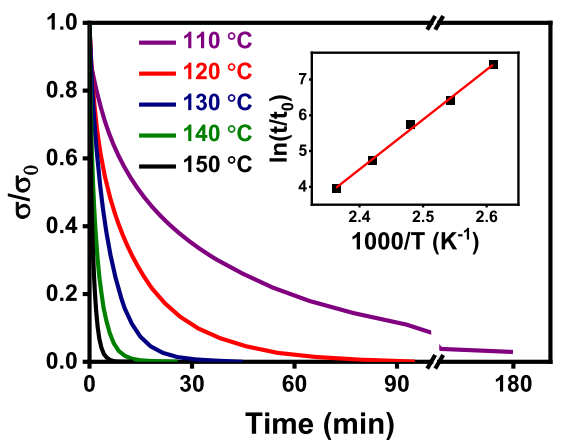

Fig. 2 Dynamic property of thiourea network. a The exchange reaction of the model compound. $\mathbf{b}$ Exchange kinetics of the model compound. $\mathbf{c}$ Isostrain stress-relaxation curves of polymer networks containing different amounts of the hindered thiourea bond (strain: $20 \%$; temperature: $130{ }^{\circ} \mathrm{C}$ ). $\mathbf{d ~ M e c h a n i c a l ~}$ properties of polymer networks with different amounts of the hindered thiourea bond. e Isostrain stress-relaxation curves of the polymer network with $21 \%$ of the hindered thiourea bond at different relaxation temperatures (strain: 20\%).

time of 0 ), the reprocessed material has a modulus lower than that of the original material highlighted as the light red rectangle. Conditioning for around 0.5 to $1 \mathrm{~h}$ would recover the modulus value. Further conditioning results in significant modulus increase until reaching a plateau value at around $18 \mathrm{~h}$. The enhancement of the strength follows a similar trend although the time for reaching plateau is shorter (around $12 \mathrm{~h}$ ). The plateau observed in Fig. 4b is mainly due to the fact the destructive thermal degradation is offset by the constructive oxidation. Upon further oxidation for $72 \mathrm{~h}$, the material does become noticeably more brittle. In accordance with the mechanical change, the peak corresponding to urea $\left(1640 \mathrm{~cm}^{-1}\right)$ is progressively enhanced in the FTIR spectra during the conditioning step in air (Fig. 4c). To quantify the hindered thiourea to urea conversion, a network sample is synthesized with its urea and thiourea contents equal to that of the above network assuming that all the hindered thiourea are converted into urea in the conditioning step. This sample is used as a reference for FTIR analysis of the reprocessed and conditioned samples (Supplementary Fig. 9). The results show that the conversion from the hindered thiourea to urea upon conditioning (Fig. 4d) follows a similar trend with the mechanical property (Fig. 4b, Supplementary Fig. 8), verifying the molecular mechanism.

To highlight the uniqueness of the oxidation enhanced strengthening, a polyurea sample with a similar network structure is subjected to reprocessing at $180^{\circ} \mathrm{C}$, followed by oxidation in air at $140^{\circ} \mathrm{C}$ for $4 \mathrm{~h}$. As expected, the mechanical performance of the polyurea deteriorates markedly after reprocessing (Supplementary Fig. 10).
The oxidation-induced strengthening is effective after repeated reprocessing. The same polythiourea sample can undergo multiple reprocessing cycles with a conditioning time of $4 \mathrm{~h}$. Figure $4 \mathrm{e}$ shows that both the modulus and strength are progressively increased in the first two reprocessing cycles. The photographic images in Fig. $4 \mathrm{f}$ provide visual evidence of the modulus enhancement in the first two cycles. After the third reprocessing cycle, the mechanical performance is declined, but still higher than that of the original sample.

From a practical standpoint, a reprocessed material only has to maintain the original mechanical properties in order to meet the requirement for its reuse. Therefore, the conditioning time can be shortened and the reprocessing cycles can exceed three. On the other hand, if upcycling is the goal, the enhancement in the mechanical properties can be finely tuned by adjusting the conditioning time. Indeed, upcycling beyond two cycles is possible by shortening the conditioning time in each cycle. This is experimentally verified with the data presented in Supplementary Fig. 11, showing four reprocessed cycles with notable mechanical improvement. We note that, the conversion of hindered thiourea into urea is accompanied with a weight loss due to the removal of the sulfur. This maximum weight loss is, however, very small (around $1.6 \mathrm{wt} \%$ ), assuming all the hindered thiourea turned into urea.

In addition, scaling up the above material is possible, but the curing time and synthesis conditions are the economical and practical factors that should be considered. The side products including imidazole generated in the synthesis and gas generated 
a
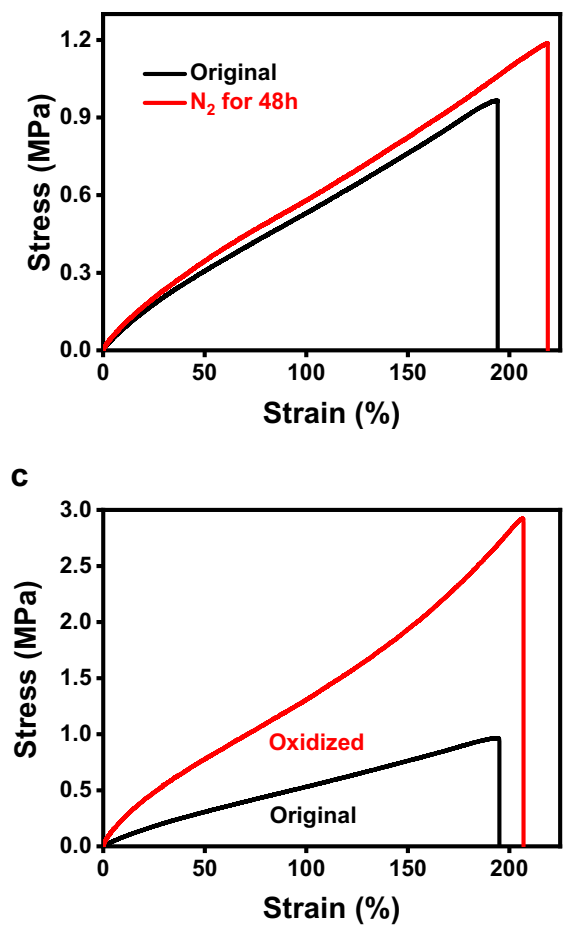

b

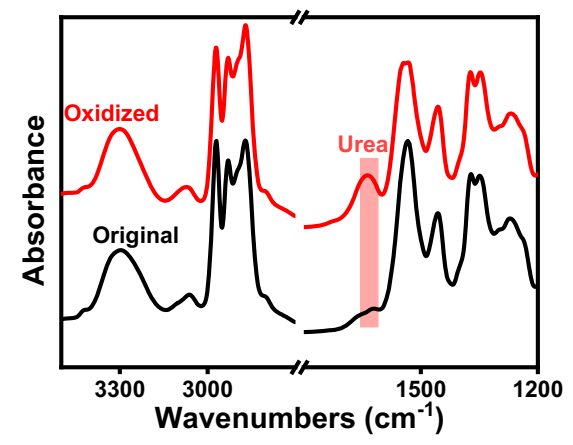

d

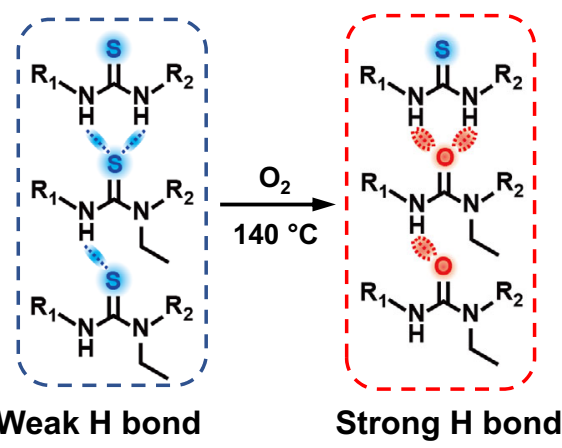

Fig. 3 Selective oxidation of the hindered thiourea bond. a The mechanical property of samples before and after thermal treatment $\left(140{ }^{\circ} \mathrm{C}, 48 \mathrm{~h}\right)$ in nitrogen flow. $\mathbf{b}$ Infrared spectra of the thiourea network (without reprocessing) before and after thermal oxidization in air for $48 \mathrm{~h}$ at $140{ }^{\circ} \mathrm{C}$. $\mathbf{c}$ Thermal oxidation (in air) induced mechanical property change of the thiourea network (without reprocessing). $\mathbf{d}$ Formation of different hydrogen bonding due to the oxidation.

during oxidation should be collected to avoid any negative environmental impact. An alternative approach to taking advantage of the material chemistry principle is to synthesize oligomers that contain the active species (thiourea bonds) and use them as starting materials for making other types of thermosets with a more conventional curing chemistry. This is a direction we are going in the future.

\section{Discussion}

Deterioration of mechanical properties after reprocessing is a major bottleneck for the sustainable uses of polymers. During reprocessing, a range of non-clean side reactions collectively contribute to the performance drop. While some of them can be mitigated (e.g., oxidation), the real challenge is to contain all of major ones given their random nature. This work reveals the dynamic characteristics of thiourea bonds, which enables the reprocessing of the network. More importantly, it utilizes the unique oxidation chemistry of hindered thiourea bonds as a mechanism for compensating the performance drop. This strategy is effective regardless of what side reactions may happen during the reprocessing. Although the upcycling is not unlimited, the materials embedded with thiourea bonds do achieve mechanical enhancement within certain reprocessing cycles. The intrinsic strengthening effect can be potentially extended beyond the thiourea chemistry. The work overall points to a promising direction in designing reprocessable polymers with markedly improved lifetime.

\section{Methods}

Materials. The chemicals used were thiocarbonyldiimidazole (TCDI, Aladdin, 95\%), poly (propylene glycol) bis (2-aminopropyl ether) (D400, Sigma Aldrich, $\mathrm{Mn} 400$ ), trimethylolpropane tris [poly (propylene glycol), amine terminated] ether (T440, Sigma Aldrich, Mn 440), N,N'-diethyl-1,6-diaminohexane (DDAH
TCI, 97\%), N, $N^{\prime}$-carbonyldiimidazole (Aladdin, 97\%), isopropyl isothiocyanate (TCI, 98\%), $N$-ethylbutylamine (TCI, 99\%), $N, N^{\prime}$-diisopropylthiourea (TCI, 99\%), hexylamine (TCI, 99\%), isopropyl isocyanate (TCI, 98\%), di-tert-butylbipheny (TCI, 98\%), benzyl isothiocyanate (TCI, 98\%), dimethylformamide (Sinopharm, 99\%), tetrahydrofuran (Sinopharm, 99\%), dichloromethane (Sinopharm, 99\%), toluene (Sinopharm, 99\%), ethyl acetate (Sinopharm, 99\%), acetone (Sinopharm, 99\%), ethanol (Sinopharm, 99\%), ether (Sinopharm, 99\%), hexane (Sinopharm, 99\%), sodium hydroxide (Sinopharm, 99\%), hydrochloric acid (Sinopharm, 38\% in water). All chemicals were used as received.

Synthesis of the thiourea networks. Based on the formulation provided in Supplementary Table 1, D400 and DDAH were dissolved in $2 \mathrm{~mL}$ of dimethylformamide. T440 and TCDI were introduced afterward. The mixture was stirred at room temperature until thiocarbonyldiimidazole was fully dissolved. The transparent solution was then poured into a Teflon mold and thermally cured at $80^{\circ} \mathrm{C}$ for $6 \mathrm{~h}$ under nitrogen flow. The sample was post-cured at $140^{\circ} \mathrm{C}$ for $12 \mathrm{~h}$ under nitrogen flow, yielding a yellow transparent thin film (with the thickness controlled at about $0.5 \mathrm{~mm}$ ). The imidazole byproduct was removed by the nitrogen flow and then entered to a cooling equipment. During the cooling process, imidazole crystallized into solid for easy recovery.

Thiourea network reprocessing. In a typical experiment, the polymer film was cut into small pieces, put into a 0.5 -mm-thick iron mold, and pressed for $30 \mathrm{~min}$ at $140^{\circ} \mathrm{C}$ under $4 \mathrm{MPa}$. The reformed polymer film was taken out the mold and kept in an oven (air environment) at $140^{\circ} \mathrm{C}$ for conditioning to yield a reprocessed sample. The above process was repeated for a subsequent reprocessing cycle.

Isostrain stress relaxation experiments. All the tests were performed using a DMA Q800 (TA instruments) in the "stress relaxation" model. In a typical test, the thin film sample was heated to the target temperature and stretched to $20 \%$ strain. The stress in the sample was monitored and recorded while the strain was maintained constant.

Tensile test. The samples used for testing were cut into rectangular shapes $(10 \times 3 \times 0.5 \mathrm{~mm})$. All tensile tests were carried out using a Zwick/Roell Z005 tensile machine. The experiments were conducted at $25^{\circ} \mathrm{C}$ at a strain rate of $10 \mathrm{~mm} / \mathrm{min}$. 
a

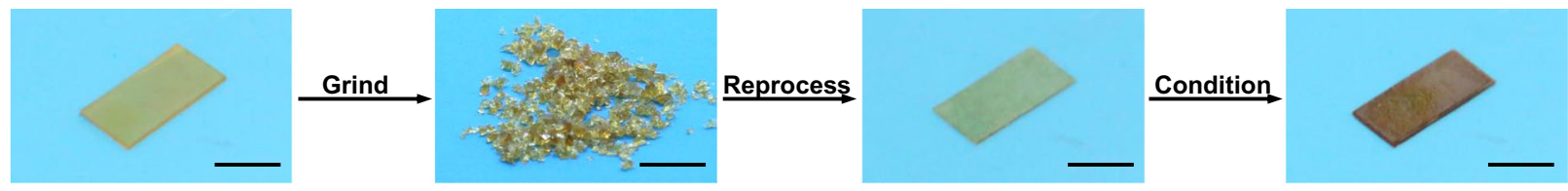

b

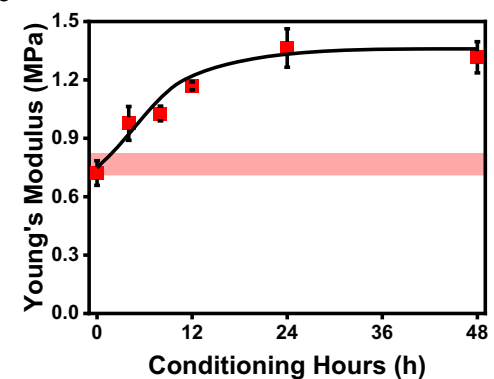

d

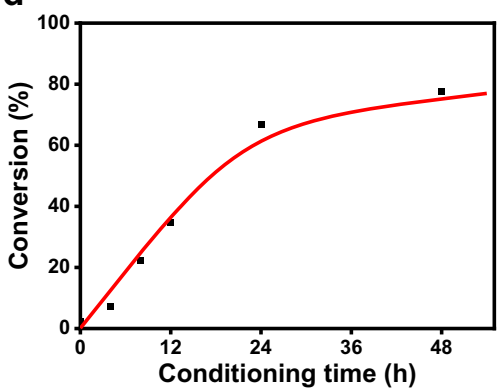

C

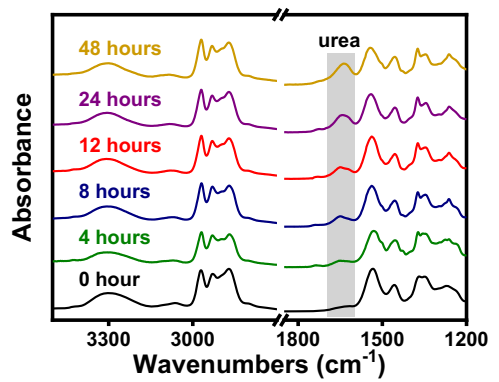

e

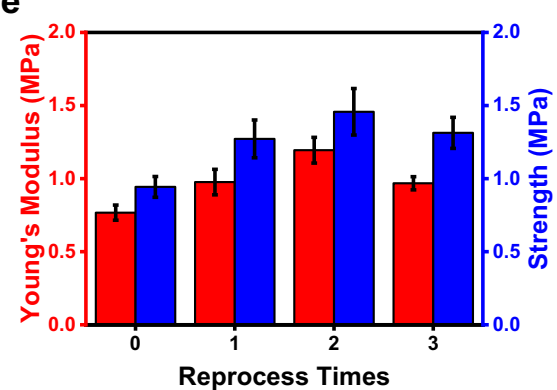

f

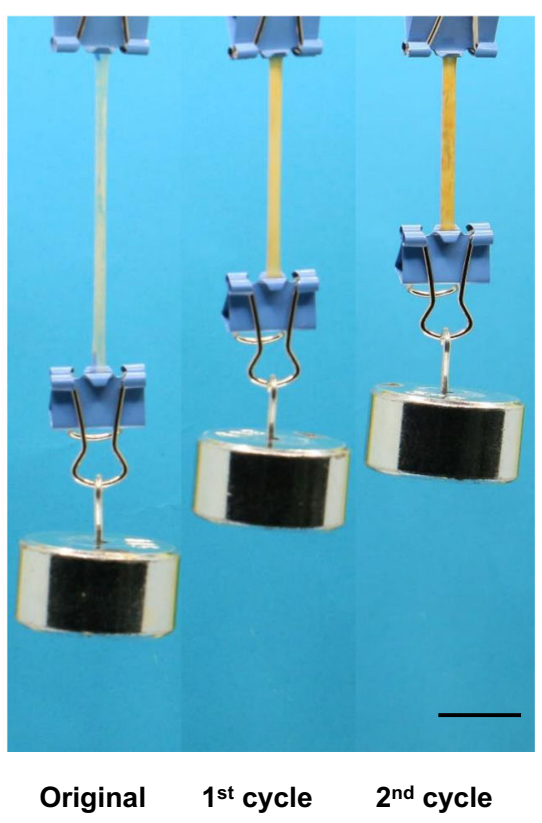

Fig. 4 Network reprocessing and enhancement in mechanical properties. a Photographic images of the sample at different stages during the reprocessing. b Change in Young's modulus of the reprocessed samples with different conditioning time in air. c FTIR change of the reprocessed samples with different conditioning time (in air). $\mathbf{d}$ Hindered thiourea conversion of the reprocessed samples with different conditioning time. e Change in Young's modulus and strength at different reprocessing cycles. For each reprocessing cycle, the conditioning time is $4 \mathrm{~h}$. $\mathbf{f}$ Photographic images of the thiourea sample with different reprocessing cycles under a constant load of $50 \mathrm{~g}$. The sample size without the external load is $15 \times 3 \times 0.5 \mathrm{~mm}$. All scale bars are $1 \mathrm{~cm}$.

Other analyses. Gas chromatography analyses were carried out using an Agilent 6890 machine using di-tert-butylbipheny as the internal standard and tetrahydrofuran as the solvent. Infrared spectrum (FTIR) analyses were conducted with thin film samples using Nicolet5700 in attenuated total reflection (ATR) mode. Temperature ramping FTIR analyses were conducted in transmission mode. HPLC-MS analyses were performed using Agilent 6545 with THF as the solvent. All the ${ }^{1} \mathrm{H}-\mathrm{NMR}$ analyses were conducted in $\mathrm{CDCl}_{3}$ with a Bruker AVANCE III $(500 \mathrm{MHz})$ machine.

\section{Data availability}

All data that support the findings of this study are available from the corresponding author on reasonable request.

Received: 1 August 2021; Accepted: 8 December 2021; Published online: 19 January 2022

\section{References}

1. Jambeck, J. R. et al. Plastic waste inputs from land into the ocean. Science 347, 768-771 (2015).

2. Garcia, J. M. \& Robertson, M. L. The future of plastics recycling. Science 358, 871-872 (2017).

3. Zheng, N., Xu, Y., Zhao, Q. \& Xie, T. Dynamic covalent polymer networks: a molecular platform for designing functions beyond chemical recycling and self-healing. Chem. Rev. 121, 1716-1745 (2021).

4. Scheutz, G. M., Lessard, J. J., Sims, M. B. \& Sumerlin, B. S. Adaptable crosslinks in polymeric materials: resolving the intersection of thermoplastics and thermosets. J. Am. Chem. Soc. 141, 16181-16196 (2019).
5. Taynton, P. et al. Heat- or water-driven malleability in a highly recyclable covalent network polymer. Adv. Mater. 26, 3938-3942 (2014).

6. Trovatti, E., Lacerda, T. M., Carvalho, A. J. F. \& Gandini, A. Recycling tires? Reversible crosslinking of poly(butadiene). Adv. Mater. 27, 2242-2245 (2015).

7. Luzuriaga, A. R. et al. Epoxy resin with exchangeable disulfide crosslinks to obtain reprocessable, repairable and recyclable fiber-reinforced thermoset composites. Mater. Horiz. 3, 241-247 (2016).

8. Shieh, P. et al. Cleavable comonomers enable degradable, recyclable thermoset plastics. Nature 583, 542-547 (2020).

9. Yu, S. et al. Catalyst-free metathesis of cyclic acetals and spirocyclic acetal. ACS Macro Lett. 9, 1143-1148 (2020).

10. Fang, Z., Zheng, N., Zhao, Q. \& Xie, T. Healable, reconfigurable, reprocessable thermoset shape memory polymer with highly tunable topological rearrangement kinetics. ACS Appl. Mater. Interfaces 9, 22077-22082 (2017).

11. Cromwell, O. R., Chung, J. \& Guan, Z. Malleable and self-healing covalent polymer networks through tunable dynamic boronic ester bonds. J. Am. Chem. Soc. 137, 6492-6495 (2015).

12. Zhang, Y. et al. Malleable and recyclable poly(urea-urethane) thermosets bearing hindered urea bonds. Adv. Mater. 28, 7646-7651 (2016).

13. Billiet, S. et al. Triazolinediones enable ultrafast and reversible click chemistry for the design of dynamic polymer systems. Nat. Chem. 6, 815-821 (2014).

14. Jin, K., Li, L. \& Torkelson, J. M. Recyclable crosslinked polymer networks via one-step controlled radical polymerization. Adv.Mater. 28, 6746-6750 (2016).

15. Obadia, M. M., Mudraboyina, B. P., Serghei, A., Montarnal, D. \& Drockenmuller, E. Reprocessing and recycling of highly cross-linked ionconducting networks through transalkylation exchanges of C-N bonds. J. Am. Chem. Soc. 137, 6078-6083 (2015).

16. Lu, Y. X., Tournilhac, F., Leibler, L. \& Guan, Z. Making insoluble polymer networks malleable via olefin metathesis. J. Am. Chem. Soc. 134, 8424-8427 (2012).

17. Sheppard, D. T. et al. Reprocessing postconsumer polyurethane foam using carbamate exchange catalysis and twin-screw extrusion. ACS Cent. Sci. 6, 921-927 (2020). 
18. Wang, Z. et al. Dynamic covalent urea bonds and their potential for development of self-healing polymer materials. J. Mater. Chem. A 7, 15933-15943 (2019).

19. Zheng, P. \& McCarthy, T. J. A surprise from 1954: siloxane equilibration is a simple, robust, and obvious polymer self-healing mechanism. J. Am. Chem. Soc. 134, 2024-2027 (2012).

20. Montarnal, D., Capelot, M., Tournilhac, F. \& Leibler, L. Silica-Like malleable materials from permanent organic networks. Science 334, 965-968 (2011).

21. García, J. M. et al. Recyclable, strong thermosets and organogels via paraformaldehyde condensation with diamines. Science 344, 732-735 (2014).

22. Wang, C. et al. Recyclable and repolymerizable thiol-X photopolymers. Mater. Horiz. 5, 1042-1046 (2018).

23. Yu, K., Shi, Q., Dunn, M. L., Wang, T. \& Qi, H. J. Carbon fiber reinforced thermoset composite with near $100 \%$ recyclability. Adv. Funct. Mater. 26, 6098-6106 (2016).

24. Li, L., Chen, X. \& Torkelson, J. M. Reprocessable polymer networks via thiourethane dynamic chemistry: recovery of cross-link density after recycling and proof-of-principle solvolysis leading to monomer recovery. Macromolecules 52, 8207-8216 (2019).

25. Qin, B. et al. Tough and multi-recyclable cross-linked supramolecular polyureas via incorporating noncovalent bonds into main-chains. Adv. Mater. 32, 2000096 (2020)

26. Ying, H., Zhang, Y. \& Cheng, J. Dynamic urea bond for the design of reversible and self-healing polymers. Nat. Commun. 5, 3218 (2014).

27. Yanagisawa, Y., Nan, Y., Okuro, K. \& Aida, T. Mechanically robust, readily repairable polymers via tailored noncovalent cross-linking. Science 359, 72-76 (2018).

28. Tian, T., Hu, R. \& Tang, B. Room Temperature one-step conversion from elemental sulfur to functional polythioureas through catalyst-free multicomponent polymerizations. J. Am. Chem. Soc. 140, 6156-6163 (2018).

29. Zhang, L. \& Rowan, S. J. Effect of sterics and degree of cross-linking on the mechanical properties of dynamic poly(alkylurea-urethane) networks. Macromolecules 50, 5051-5060 (2017).

30. Ding, H., Zhao, B., Mei, H., Li, L. \& Zheng, S. Transformation of commodity poly(hydroxyether of bisphenol A) into vitrimers via post crosslinking with hindered urea bonds. Chinese J. Polym. Sci. 38, 915-920 (2020).

31. Sahu, S., Sahoo, P. R., Patel, S. \& Mishra, B. K. Oxidation of thiourea and substituted thioureas: a review. J. Sulphur Chem. 32, 171-197 (2011).

32. Stefaniu, C. et al. Rigid urea and self-healing thiourea ethanolamine monolayers. Langmuir 31, 1296-1302 (2015).

\section{Acknowledgements}

We thank National Natural Science Foundation of China (No. 52033009, No. 21625402, No. 52003232), China National Postdoctoral Program for Innovative Talents
(BX20190294), and China Postdoctoral Science Foundation (2020M681821) for the support of this work. We thank Mrs. Jing He and Mrs. Li Xu for their assistance in performing spectrum and thermomechanical analyses at State Key Laboratory of Chemical Engineering (Zhejiang University).

\section{Author contributions}

N.Z., T.X., and H.F. conceived the idea. N.Z. and H.F designed the experiments. H.F. carried out the experiment. W.P., C.N., and H.S. participated in visualizing the mechanism. The project was supervised by T.X., N.Z., and Q.Z. T.X., N.Z., and H.F wrote the manuscript. All authors participated in result analysis and discussion.

\section{Competing interests}

The authors declare no competing interests.

\section{Additional information}

Supplementary information The online version contains supplementary material available at https://doi.org/10.1038/s41467-022-28085-2.

Correspondence and requests for materials should be addressed to Ning Zheng.

Peer review information Nature Communications thanks the anonymous reviewer(s) for their contribution to the peer review of this work.

Reprints and permission information is available at http://www.nature.com/reprints

Publisher's note Springer Nature remains neutral with regard to jurisdictional claims in published maps and institutional affiliations.

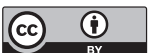

Open Access This article is licensed under a Creative Commons Attribution 4.0 International License, which permits use, sharing, adaptation, distribution and reproduction in any medium or format, as long as you give appropriate credit to the original author(s) and the source, provide a link to the Creative Commons license, and indicate if changes were made. The images or other third party material in this article are included in the article's Creative Commons license, unless indicated otherwise in a credit line to the material. If material is not included in the article's Creative Commons license and your intended use is not permitted by statutory regulation or exceeds the permitted use, you will need to obtain permission directly from the copyright holder. To view a copy of this license, visit http://creativecommons.org/ licenses/by/4.0/.

(C) The Author(s) 2022 